\title{
Voltage-Based Droop Control of Renewables to avoid On-Off Oscillations caused by Overvoltages
}

\author{
T. L. Vandoorn, J. D. M. De Kooning, B. Meersman and L. Vandevelde \\ Electrical Energy Laboratory (EELAB), Department of Electrical Energy, Systems and Automation (EESA), \\ Ghent University, Sint-Pietersnieuwstraat 41, B-9000 Ghent, Belgium, Phone: +32 926434 22, \\ e-mail: Tine.Vandoorn@UGent.be
}

\begin{abstract}
To achieve the environmental goals set by many governments, an increasing amount of renewable energy, often delivered by distributed generation (DG) units, is injected in the electrical power system. Despite the many advantages of DG, this can lead to voltage problems, especially in times of a high local generation and a low local load. The traditional solution is to invest in more and stronger lines, which could lead to massive investments to cope with the huge rise of DG connection. Another common solution is to include hard curtailment, thus, on/off control of DG units. However, hard curtailment potentially leads to on-off oscillations of DG and a high loss of the available renewable energy as storage is often not economically viable. To cope with these issues, applying a grid-forming control in grid-connected DG units is studied in this paper. The voltagebased droop control, that originally has been developed for power sharing in islanded microgrids, enables an effective way for soft curtailment without communication. The power changes of the renewable energy sources are delayed to more extreme voltages compared to those of the dispatchable units. This restricts the renewable energy loss and avoids on-off oscillations.
\end{abstract}

Index Terms-distributed generation, droop controllers, on-off control, voltage control, voltage oscillations

\section{INTRODUCTION}

Nowadays, fossil fuel is the main source of energy, leading to environmental, political and economical concerns. Renewable energy sources can provide a solution [1]. Because of the lower power density and the geographical dispersion, these technologies are mostly available as small distributed generation (DG) units. DG offers many advantages, such as enabling an increased usage of environmental friendly resources and postponement of investments in new transmission systems and large-scale generators [2]. Their positioning near the consumers can lead to a reduction of transmission and distribution losses. However, the growing share of DG units also has several implications on the operation and planning of the electrical network [3]-[6].

To deal with the increased penetration of DG, a coordinated approach for integrating DG is required. Therefore, the microgrid concept has been introduced [7], [8]. Microgrids are likely to play a key role in the evolution of the smart grid [9], [10] as it is expected that the smart grid will emerge as a system of integrated smart microgrids [11]. A key advantage is that the microgrid appears to the power network as a controllable entity [12]. Microgrids can operate both in islanded and gridconnected mode [13] and address technical, economical and environmental concerns in modern power systems [14]. As most DG units are connected to the electrical network via a voltage-source inverter (VSI), microgrid control involves the control of these inverters [15]. In the grid-connected mode, currently, the DG units are equipped with a grid-following control that is generally current-controlled [16]-[18]. The primary function of these controllers is to inject a specified amount of power into the network. In islanded microgrids, at least one grid-forming unit, which is voltage-controlled, should be present because of the lack of a utility grid. The grid-forming controllers are responsible for the voltage control (amplitude and frequency) and power sharing. A well-known and widely-used control strategy is droop-based control. Droop controllers operate without communication in order to avoid a critical information infrastructure for reliability reasons. They can be classified in either active power/grid frequency $(P / f)$ droop control [8], [19]-[21] or active power/terminal voltage $\left(P / V_{\mathrm{g}}\right)$ droop control [22], [23].

Compared to the grid-forming units in islanded microgrids, the grid-following DG units generally do not contribute in the grid support, i.e., control of voltage amplitude and frequency. Because of this passive operation of DG, local grid bottlenecks appear already today, e.g., for photovoltaic installations at the end of the lines or in areas with a high density of DG. Two central factors that restrict the available additional DG capacity in distribution networks are the voltage increase and voltage unbalance [24]. Therefore, the fit-and-forget strategy of installing DG is not a sustainable option and limits the penetration of DG. In the future, DG will need an active control to contribute in the grid support, e.g., to cooperate in the voltage control, power sharing and to provide other ancillary services such as reserve.

To provide voltage support, the conventional large power plants are equipped with reactive power/terminal voltage $\left(Q / V_{\mathrm{g}}\right)$ droop controllers. The grid-connected DG units can be equipped with analogous $Q / V_{\mathrm{g}}$ droop functions, e.g., the static voltage support of SMA inverters [25]. However, in lowvoltage networks, voltage support through reactive power is generally inefficient as the grid voltage is linked with the active power, not the reactive power, because of the predominantly resistive lines. Hence, large amounts of $Q$ are required to influence the voltage. $P / V_{\mathrm{g}}$ controllers are more effective and straightforward to provide the voltage support in low-voltage networks.

The conventional on-off control (hard curtailment) is compared with soft curtailment. In [26], hard curtailment is com- 
pared with communication-based soft curtailment, relying on a smart metering infrastructure. In the current paper, soft curtailment is analysed in a fast-acting primary control scheme, based on droops that do not depend on communication. On-off control can lead to power quality degradation (large voltage and current transients), loss of available renewable energy and problems with the inverters (damage or accelerated ageing). Therefore, soft curtailment needs to be considered. An easy way to include soft curtailment in the DG units is by implementing a $P / V_{\mathrm{g}}$ droop controller. The current-controlled (grid-following) DG units can be equipped with $P / V_{\mathrm{g}}$ droops. An inherent disadvantage of grid-following units is that they need voltage tracking, generally by using a phase-locked loop (PLL), for the synchronisation of the unit to the grid. The $P / V_{\mathrm{g}}$ droop controller also depends on voltage tracking to extract the voltage amplitude. Grid-forming controllers in gridconnected DG units can be implemented as well. This is analogous to the transmission network in which the large central generators are grid-forming. In this paper, the voltagebased droop (VBD) control, that is developed for grid-forming units in islanded microgrids [27], is used for voltage control by DG units in grid-connected networks for two reasons. Firstly, the VBD control engages renewables in the voltage support while delaying the changes of the active power of the renewables to more extreme voltages compared to those of dispatchable DG units. Secondly, it presents a primary control operating without the need for inter-unit communication nor voltage tracking for synchronisation. It is shown that the VBD control is effective to avoid on-off oscillations (analogous to the $P / V_{\mathrm{g}}$ droops). With $\mathrm{VBD}$ control, the renewables also take part in the voltage control, but with a lower priority of power changes compared to that of the dispatchable DG units (in contrast to the $P / V_{\mathrm{g}}$ droops). In this way, the energy capturing of renewables is increased.

The paper is structured as follows. In $\S$ II an overview of the on-off and VBD control principles is given. In $\S$ III these controllers are compared with respect to the renewable energy capturing, over-voltage occurrence and on-off oscillations compared to the on-off control of DG units. To further increase the renewable energy capturing, a $Q / V$ droop is added to the VBD control strategy. In $\S$ IV, some examples are studied to compare these methods with respect to the onoff oscillations, and voltage quality. The delivered energy with the different controllers is calculated, showing that onoff oscillations significantly reduce this energy. By using the VBD control and the VBD control with the extension of $Q / V$ droops, the renewable energy capturing is significantly increased.

\section{Voltage CONTROL BY MEANS OF DG UNITS}

Currently, most DG units deliver an amount of power to the electrical network that is independent of the state of the network. This input power is determined solely by the energy source, e.g., maximum power point tracking in case of photovoltaic panels and wind turbines, or heat as primary driver in combined heat and power (CHP) units. Also, most units are current-controlled in a grid-following control strategy. In this paper, grid-forming and grid-following control strategies in grid-connected microgrids or utility feeders are considered.

\section{A. Grid-following unit with on-off control}

Firstly, conventional grid-following controllers with on-off control as depicted in Fig. 1 are considered. The phase angle ( $\theta$ in Fig. 1) of the reference current is obtained by tracking the terminal voltage by using a PLL. The current amplitude is obtained by a dc-link voltage controller keeping the dc-link voltage constant, while the input dc-power is independent of the state of the network. The on-off controller shuts down the DG unit in case the voltage exceeds a predefined value (e.g., $\left.110 \% V_{\mathrm{g}, \text { nom }}\right)$, called the critical voltage.

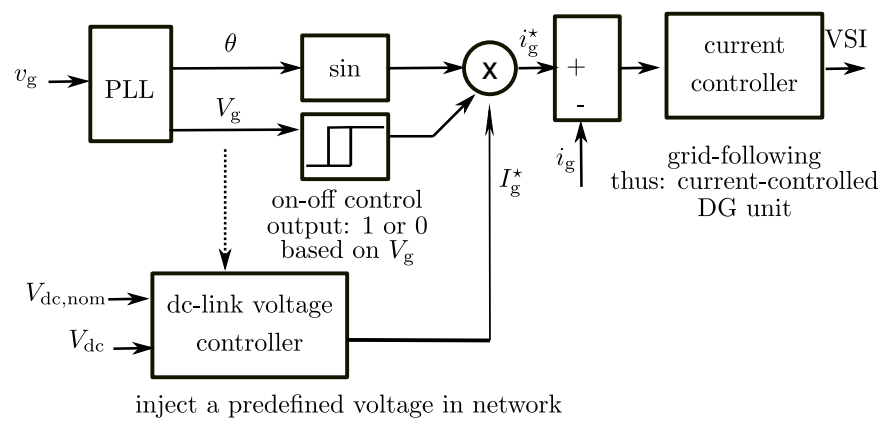

if output on-off controller $=1: P$ not dependent on network state if output on-off controller $=0: P=0 \mathrm{~W}$ (or: changed by storage, $\cdots$ )

Fig. 1. Grid-following unit with on-off control based on the terminal voltage

Instead of implementing a $100 \%$ change of active power (on-off) in case of over or under voltage, the delivered active power of the unit can be dependent on the local voltage $\left(P\left(V_{\mathrm{g}}\right)\right)$. For this, a grid-following $P / V_{\mathrm{g}}$ droop control strategy, that does not require a communication infrastructure, can be used.

\section{B. Grid-forming unit with on-off control}

Next to the conventional grid-following controllers, it is shown that a basic grid-forming controller is possible in the grid-connected DG units as well. With grid-connected DG units, DG units that are connected to the utility network or a grid-connected microgrid are meant, not units connected to an islanded microgrid. In the control of the VSIs ac-side, always four parameters are present: the amplitude and phase angle of the terminal voltage and current. Two of these parameters can be controlled. Whereas the grid-following controllers are current-controlled (amplitude and phase of the current), the grid-forming controllers are voltage-controlled (amplitude and phase of the ac voltage), as is shown in Fig. 2 A PLL for voltage tracking is not required, which simplifies the control algorithm. The units are synchronized by the $Q / f$ droop controllers, which measure the reactive power and change the frequency, thus phase angle, accordingly. This relies on the natural linkage between $Q$ and phase angle differences in resistive networks.

Like in the grid-following units, these DG units can easily limit the injected current as their voltage control loop is often composed of an outer voltage and an inner current control 
loop. The grid-following DG units are mostly equipped with a power-factor-one controller. In the grid-forming controllers, this is inherently present as well. The reason is that the $Q / f$ droop control operates at $Q_{\text {nom }}$ in case $f=f_{\text {nom }}$. The conventional generators force the grid frequency to its nominal value through secondary control. As $Q_{\text {nom }}$ in most DG units equals zero, this means that these units operate at power-factorone. This can be altered by changing $Q_{\text {nom }}$, for example in a secondary controller driven by smart grid communication and control.

These controllers also inject a predefined amount of active power in the network and operate with on-off control reacting on over-voltages. Like in the grid-following control, $P / V$ control can also be included in these units.

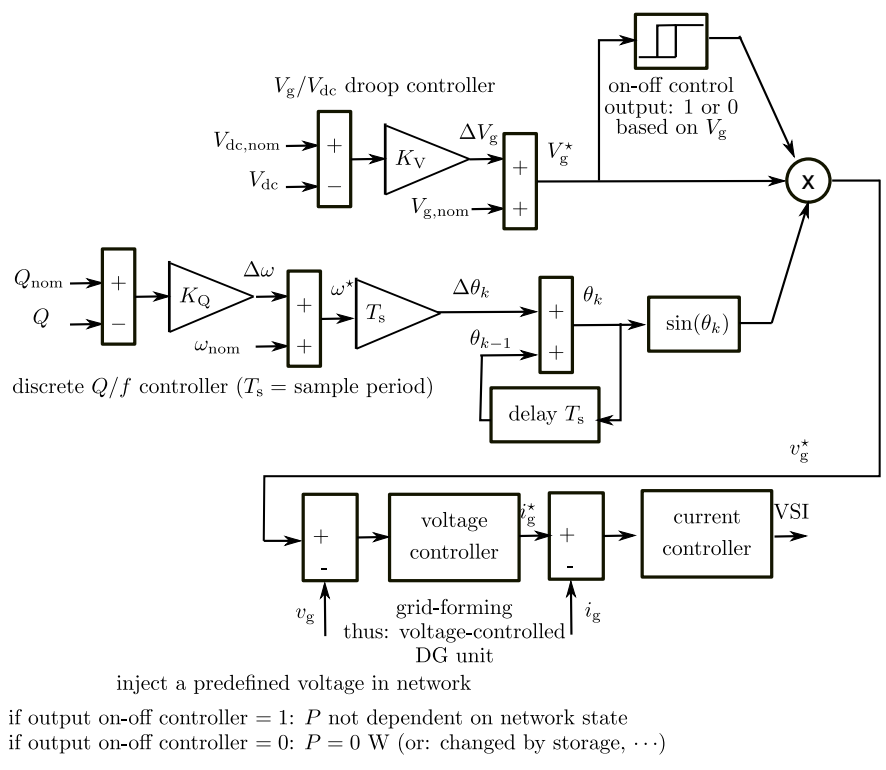

Fig. 2. Grid-forming unit with on-off control based on the terminal voltage

\section{Grid-forming unit with voltage-based droop control}

The voltage-based droop (VBD) control strategy, which has been presented for islanded microgrids in [27], [28], is applied in the grid-connected units to change their $P$ based on the network state. The active power can be altered by changing the input from the energy source (biomass supply, changing the wind turbines pitch angle, deviating from maximum power point (MPP) in a photovoltaic system), by using energy storage or shifting the local load. The control principles of the VBD controllers are summarized in Figs. 3 and 4 . Fig. 3 shows the cascaded operation of the $V_{\mathrm{g}} / V_{\mathrm{dc}}$ droop controller, enabling ac and dc-side balancing of the inverter, and the $P_{\mathrm{dc}} / V_{\mathrm{g}}$ droop controller for the voltage limiting without inter-unit communication. It also shows a so-called constant-power band with a width dependent on the nature of the energy source, which is clarified in Fig. 4. Renewable energy sources with large constant-power bands are triggered by more extreme voltages to alter their delivered power compared to the units with smaller constant-power bands. Communication is not required for this different reaction of the units on the terminal voltage. The $P_{\mathrm{dc}} / V_{\mathrm{g}}$ droop is generic and can be adapted

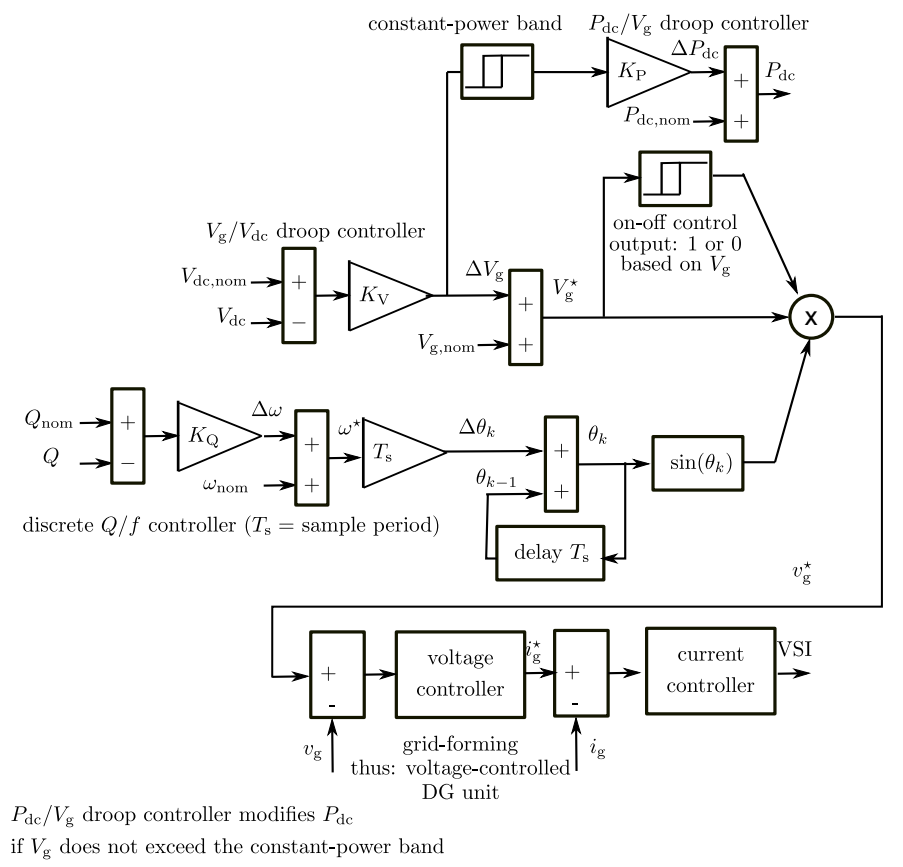

Fig. 3. Grid-forming unit with VBD control

according to the nature of the energy source. For a photovoltaic system for example, in Fig. 4(c), assuming operation in the $\operatorname{MPP}\left(P_{\mathrm{dc}, \text { nom }}=P_{\mathrm{MPPT}}\right)$, the system cannot by itself respond to under-voltage conditions. Hence, in this case, the on-off controller shuts down the unit. By deviating from the MPP, the panel can act on over-voltages (which are more probable than the under-voltages in case of a high renewable input). The constant-power bands for over-voltage should be set below the voltage limit, e.g., $b=8 \%$ for a voltage limit of $10 \%$. Dispatchable units, where $P_{\mathrm{dc}, \text { nom }}$ can be agreed upon in the energy markets, can act on under-voltage conditions by increasing their fuel intake as Fig. 4(a) suggests. An advantage of the VBD control is that the rms voltage does not need to be extracted from the measured terminal voltage as it is the output of the $V_{\mathrm{g}} / V_{\mathrm{dc}}$ droop controller.

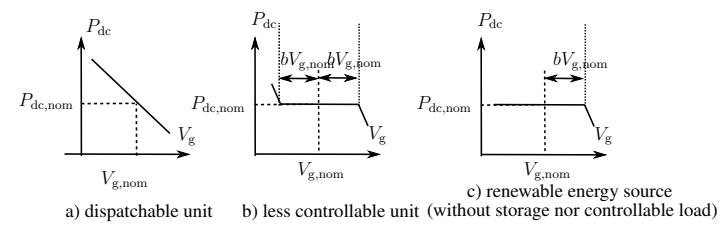

Fig. 4. Constant power bands, with a width $h=2 b$, of dispatchable versus less-dispatchable DG units

\section{EFFECT OF THE CONTROL ALGORITHMS ON ON-OFF OSCILLATIONS}

\section{A. Grid-following controller}

1) On-off control: In the grid-following control, a PLL tracks the terminal voltage of the DG units. The PLL calculates the phase angle $\theta$ of the voltage and the rms value $\left(V_{\mathrm{g}}\right)$. In order to inject a certain amount of power $P$ (e.g., $P$ 
determined by MPPT) into the microgrid, the reference current is calculated from:

$$
i_{\text {ref }}(\mathrm{t})=\sqrt{2} \frac{P}{V_{\mathrm{g}}} \sin (\theta)
$$

Here, a power-factor-one control is used ( $Q=0 \mathrm{VAr})$. The output power $P$ equals $P_{\mathrm{dc}, \text { nom }}$, except when the unit is turned off by its on-off controller, then, $P=0 \mathrm{~W}$.

The on-off control of the DG units is based on a hysteresis function. It turns off the DG unit when its terminal voltage exceeds a certain voltage and turns it back on when the voltage falls back to a lower voltage, and vice versa for under-voltage conditions. It is clear that when the DG unit turns off due to over-voltage, the voltage at the unit's terminals will drop. If the hysteresis function is not chosen properly, the voltage can drop below the turn-on voltage, turning the unit back on, which may lead to oscillations. An example of a measured voltage oscillation problem is depicted in Fig. 5. The first DG unit clearly shows an on-off oscillation. A proper choice of the parameters of the hysteresis function can solve the problem. However, as the network varies dynamically, this is difficult to achieve. Also, for all the DG units, the hysteresis function needs to be set according to the network characteristic, which is time consuming and not always fair (e.g., units at the end of the line are likely to turn off more than other ones).

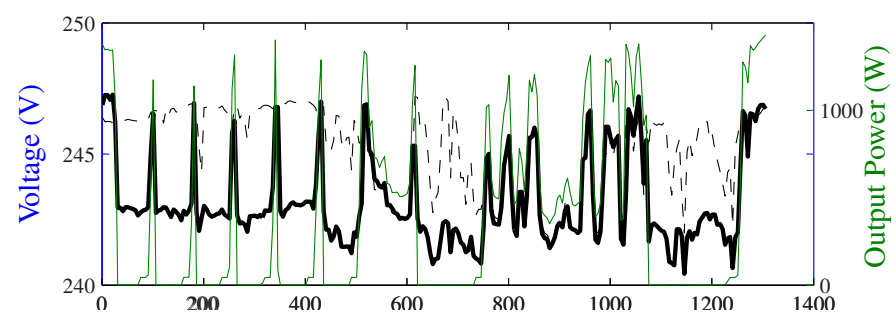

Fig. 5. On/off control of DG leading to grid-oscillations: measurements of $\mathrm{PV}$ panels in Oostende, Belgium $(-=\mathrm{DG}$ unit $1,---=\mathrm{DG}$ unit 2, $\left.V_{\mathrm{PCC}}\right)$

2) Trial strategy to limit power decrease: In practice, the DG units turn off during a specified amount of time, e.g., 30 min, in case the number of on-off swings in a certain time period is higher than a threshold value. However, this may lead even more to renewable energy loss or over-sizing of the storage facilities. The reason is that often the DG unit should not have been turned off entirely in order to solve the voltage problem. A solution to avoid these oscillations is, hence, to change the hysteresis function's parameters online, for example, based on a trial and error strategy. Analogously, the percentage with which the DG units lower their output power, instead of turning off entirely, can be set based on a strategy that is here called the trial strategy. This strategy should determine the minimum amount of power reduction to avoid oscillations while still meeting the voltage limits. In [29], this optimal power change is determined by a smart grid algorithm. However, here, the controller should operate without communication as it involves a primary control such that optimal power cannot be determined in a straightforward manner. Therefore, the unit takes trial and error attempts by changing its power and, based on the according voltage change, searching for the minimum required power change.

In the trial strategy, when the voltage becomes too high, a drop of for example $10 \%$ with a hysteresis function is implemented. If this is not sufficient to solve the voltage problem, the drop is changed to $20 \%$, etc. This is not optimal concerning the renewable energy capturing, but avoids oscillations. For safety reasons, the controller can also start with a $100 \%$ power drop and gradually lower this until no more oscillations occur while still avoiding voltage limit violation. As this method is not practical, it is not discussed further, it is merely included to emphasize that fully turning off is not always required to avoid the voltage problem.

3) Soft curtailment: Soft curtailment in all DG units can avoid the on-off oscillations, hence, achieves higher energy capturing of the renewable energy sources. Instead of turning off entirely when a voltage limit is exceeded, the output power can gradually be lowered when the terminal voltage rises, by using a $P / V_{\mathrm{g}}$ droop controller. However, this leads to renewable energy loss. The set-point of $P$ automatically decreases with an increase of $V_{\mathrm{g}}$ even if other dispatchable DG units nearby have sufficient margin to solve the voltage problem. This is not compatible with the priority injection of renewables that should be able to inject their generated power if the power quality can be maintained by other units.

4) Other methods to avoid oscillations: The smart grid paradigm also covers mitigation of voltage problems. This is done by communicating new power set-points to the concerned DG units. However, this requires communication for the voltage control, which can reduce the reliability of the system. Hence, in this paper, it is emphasized that with respect to the robustness of the system, communication should be avoided for primary control and protection issues. However, smart grid communication, management and control systems are very interesting for secondary issues and to support the automatic controllers for a more economical and optimal operation.

In the examples below, the on-off controller (hard curtailment) is compared with the VBD control. An optimal power change can be found by using communication and a smart metering infrastructure, implemented in a slow control scheme, overlaying the fast-acting primary control schemes that are analysed in this paper.

\section{B. VBD control to avoid oscillations}

As discussed above, the $P / V_{\mathrm{g}}$ droop controller, which can be implemented in both grid-following and grid-forming DG units, avoids the on-off oscillations. However, it does not distinct between the dispatchable and less dispatchable (e.g., renewable) DG units. Either no droops are implemented in the renewables, such that the oscillation problem is not solved in places with high renewable penetration. Either droops are implemented, solving the issue that on-off oscillations can occur when one unit has a large impact on the grid voltage, by changing the output of the unit depending on the voltage. However, this leads to a significantly lower renewable energy capturing as priority of power changes should be given to dispatchable DG units. Dispatchable units should act more and first to voltage rises, while the renewable energy sources 
should act only when absolutely necessary for the voltage control of the network. Therefore, the VBD control sets an automatic priority based on the terminal voltage of the network. The output power changes of dispatchable DG units are prioritized over those of the less dispatchable ones. The automatic nature of the priority setting is crucial for the reliability of the system. Of course, smart grid features can change the settings of the VBD controller (e.g., the constantpower band width and the reference power) in an overlaying secondary control scheme.

\section{VBD control with reactive power consumption}

In [29], the required amount of reactive power $Q$ that a DG unit should consume to minimize its impact on the voltage variations is calculated. A minimal impact is achieved when the DG unit consumes (minus sign)

$$
Q=-\frac{R}{X} P,
$$

with $R$ and $X$ the line parameters and $P$ the generated active power of the DG unit. Here, a uniform distribution of load along the feeder and constant resistance and reactance per unit length are assumed. Still, a small impact remains due to the power losses associated with transport of power over the network, which are not included in (2). Here, low-voltage networks, which are mainly resistive, are considered, hence, with a high $R / X$, i.e., generally larger than three. Accordingly, such large amounts of reactive power can generally not be consumed by the generators without significant overrating. The reactive power consumed by the downstream load could be used to compensate part of the voltage rise, however, the load is generally unknown and variable in time. Therefore, a suboptimal amount of reactive power can be consumed by the generator, compromising between injecting more active power (which is analogous to limiting the impact of the unit's active power injection on the feeder voltage) and limiting the overrating of the unit.

In order to improve the capturing of renewable energy, while still avoiding oscillations and over-voltages, in this section, a $Q / V$ droop is included in the VBD control. In this way, the unit consumes reactive power when the voltage is out of the constant-power band. In Fig. 3 , the value $Q_{\text {nom }}$ (which is mostly 0 in the conventional VBD control) is dependent on $\Delta V_{\mathrm{g}}$. When the voltage is out of the constant power band $Q_{\text {nom }}$ becomes $K_{P} \Delta V_{\mathrm{g}} \sin \theta\left(\tan \theta=X / R\right.$ and $\left.K_{P}<0\right)$, otherwise $Q_{\text {nom }}$ remains zero. By using $\theta$, dependent on the line characteristics, $Q_{\text {nom }}$ is not changed in purely resistive networks $(\theta=0)$ because $Q$ consumption would have little effect on the voltage (hence, capturing of energy) as (2) reflects. Also, instead of using $\Delta V_{\mathrm{g}}$ as input of the $P_{\mathrm{dc}} / V_{\mathrm{g}}$ droop controller, $\Delta V_{\mathrm{g}} \cos \theta$ is used.

Finding the absolute optimal value of $Q$ to be consumed is not the focus of a primary control strategy. The VBD control succeeds in avoiding the on/off oscillations, while automatically giving priority to renewable injection. By changing $Q_{\text {nom }}$, its impact on the voltage is decreased, which will increase the energy capturing (more power can be injected in the network). However, the optimal amount of reactive power is, in the considered resistive networks, often too high for the inverter. To optimize the network, secondary controllers, which can use communication (e.g., concerning the downstream load), can change the settings of the primary controllers.

\section{EXAMPLES}

The controllers discussed above, i.e., on-off control, VBD control and VBD control with $Q / V$ droops, are compared with respect to the on-off oscillations and capturing of renewable energy. The grid-connected microgrid topology is depicted in Fig. 6 A typical EAXVB cable has a line impedance of 0.1$0.4 \Omega / \mathrm{km}$. The higher the line resistance, the more significant the voltage problem in the network. Therefore, line resistance values on the upper margin are considered. The utility network is modelled as a $230 \mathrm{~V} \mathrm{rms}$ and $50 \mathrm{~Hz}$ voltage source, i.e., a strong network. The DG units consist of a VSI with dcbus $\left(V_{\mathrm{dc}, \text { nom }}=700 \mathrm{~V}, C_{\mathrm{dc} \text {,nom }}=1.5 \mathrm{mF}\right)$ and an LC filter $(L=2 \mathrm{mH}, C=3 \mu \mathrm{F})$. The microgrid consists of three DG units. The VSIs are modelled upto the level of the converter switches. The ac-side current and voltage controllers in Figs.11. 2 and 3 consist of PI controllers. At the dc-side, the sources are modelled as constant-current sources, as the dc-side is not modelled in detail and relatively short time frames (transient changes) are studied. Hence, a $I_{\mathrm{dc}} / V_{\mathrm{g}}$ droop controller is used $\left(I_{\mathrm{dc}}=I_{\mathrm{dc}, \text { nom }}+K_{P}\left(V_{\mathrm{g}}-V_{\mathrm{g}, \text { nom }}\right)\right)$, which is completely analogous to the $P_{\mathrm{dc}} / V_{\mathrm{g}}$ droop controller for power sources. DG 1 has a constant $I_{\mathrm{dc}, \mathrm{nom}}$. DG 2 on the other hand has the initial $I_{\mathrm{dc}, 2, \text { nom }}$ when $t<1 \mathrm{~s}$ and $0.5 I_{\mathrm{dc}, 2, \text { nom }}$ when $t>1 \mathrm{~s}$. DG 3 has $I_{\mathrm{dc}, 3, \text { nom }}$ when $t<0.35 \mathrm{~s}, 0.5 I_{\mathrm{dc}, 3, \text { nom }}$ when $0.35<t<0.70 \mathrm{~s}$ and $1.25 I_{\mathrm{dc}, 3 \text {,nom }}$ when $t>0.70 \mathrm{~s}$. Note that the actual dc current is equal to $I_{\mathrm{dc}, \text { nom }}$ in case the on-off controllers are used. In VBD control, this value is dependent on the terminal voltage according to the $I_{\mathrm{dc}} / V_{\mathrm{g}}$ droop controller.

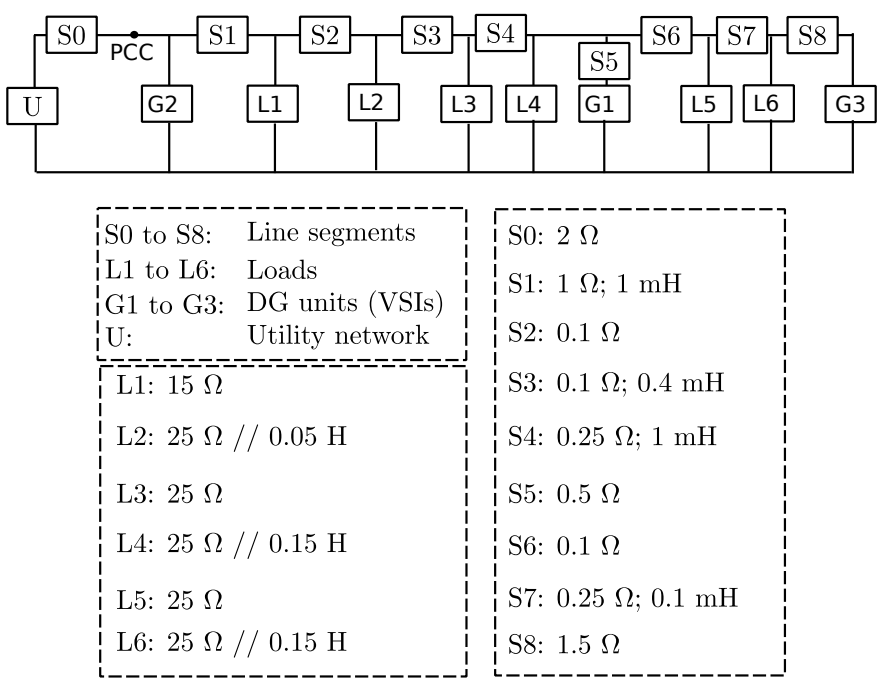

Fig. 6. The considered microgrid topology, three DG units and six loads, grid-connected

\section{A. On-off control}

The on-off control of the three DG units is based on a hysteresis function that turns off the DG unit when its terminal 
voltage exceeds $109 \% V_{\mathrm{g}, \text { nom }}$ and turns it back on when the voltage again drops below $102 \% V_{\mathrm{g}, \text { nom }}$. An important remark concerning the following figures, is that the time scales are unrealistically short. The paper does not focus on how the on/off swings occur (because this is highly dependent on the specific time constant of the DG units), but rather on whether or not they occur. The measurements and on/off control actions are assumed to be performed with the switching frequency of the units. Hence, the oscillations can also occur very fast. Of course, in practice, the oscillations follow up less quickly, firstly, because the voltage measurements can be slower, and secondly, because most units turn off for a specified amount of time after over-voltage or under-voltage occurred. This is not included in the simulations to easily compare the controllers for equal boundary conditions in a limited simulation time. The fact that this is not included in the simulations, does not interfere with the general conclusions, focussing on comparing the different strategies. The time scale (horizontal axis) can be changed based on the real turn-off time of the DG units.

In the first case, the nominal dc currents equal $5 \mathrm{~A}, 7.5 \mathrm{~A}$ and 10 A for the grid-following units DG 1, DG 2 and DG 3 respectively. The simulation results are depicted in Fig. 7 . A clear on-off oscillation of DG 3 is shown, except when $0.35<t<0.70 \mathrm{~s}$ (low $I_{\mathrm{dc}, \text { nom }, 3}$ ). When DG 3 disconnects, the grid voltage decreases significantly. The voltage falls below the $102 \% V_{\mathrm{g}, \text { nom }}$ voltage limit, such that the DG unit turns back on, etc. This is due to the high local penetration of DG units in a network with clearly resistive lines. On-off control is hence solely effective if the influence of one DG unit on the terminal voltage is not too large and the penetration of DG is sufficiently low. Therefore, this is not a sustainable situation when considering for example the European 202020 targets for a higher penetration of renewable sources in the network, which are often small low-voltage connected DG units.

A solution to avoid the large renewable energy loss can be to replace the on-off controller with the trial strategy that decreases $I_{\mathrm{dc}}$ with, for example only $30 \%$ instead of turning off entirely. Therefore, in the second case, the same relay function to control the delivered power to the network is used, but instead of turning the DG unit off in case of high voltages, a $30 \%$ dc-current change is included (here called the $70 \%$ control). Again, the nominal dc-currents of the DG units equal $5 \mathrm{~A}, 7.5 \mathrm{~A}$ and $10 \mathrm{~A}$ for DG 1, DG 2 and DG 3 respectively. The simulation results in Fig. 8 show that the oscillations are no longer present. This example illustrates that the DG unit should not have been turned off entirely in order to solve the voltage problem. However, the percentage at which the power should decrease is hard to find in an elegant and allaround manner. An overlaying, communication-based, smart grid control algorithm can help finding the optimal percentage. The latter is not an objective of the primary control strategies considered here.

It is also possible to include grid-forming controllers with the on-off functionality. In this paragraph, the VBD control is included in the DG units with constant-power band $b=\infty$. This represents the control with delivered power independent on the state of the network, but implemented in a grid-forming controller. Again, on-off control is included

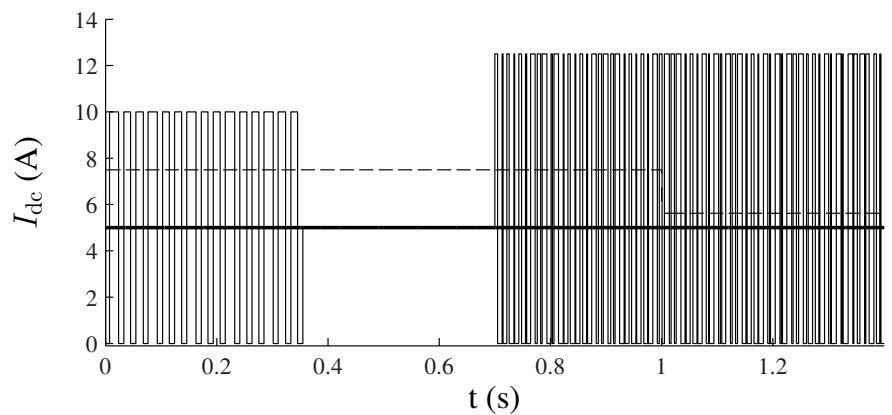

(a) Dc-input current of DG unit

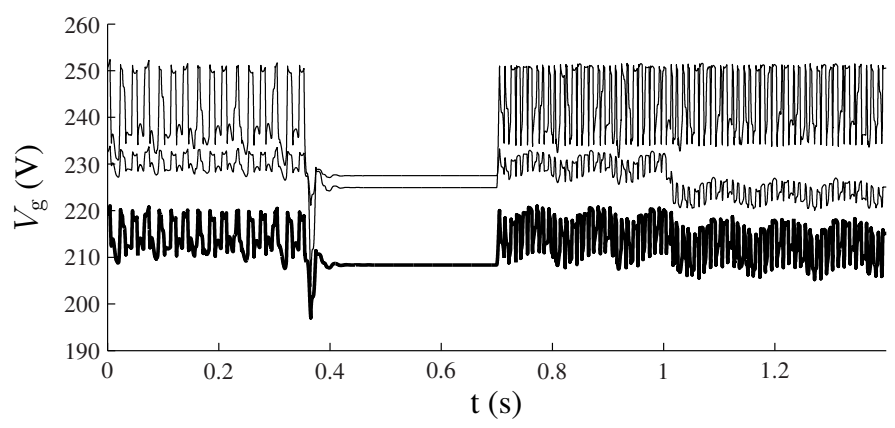

(b) Terminal voltage

Fig. 7. Grid-following on-off control, first case $I_{\mathrm{dc}, \text { nom }}: 5 \mathrm{~A}, 7.5 \mathrm{~A}$ and $10 \mathrm{~A}(-=\mathrm{DG} 1 ;----=$ DG $2 ;-=$ DG 3 )

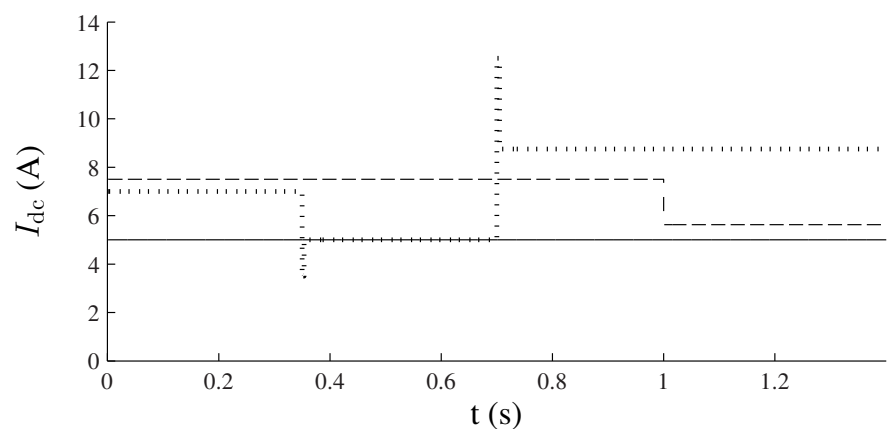

(a) Dc-input current of DG unit

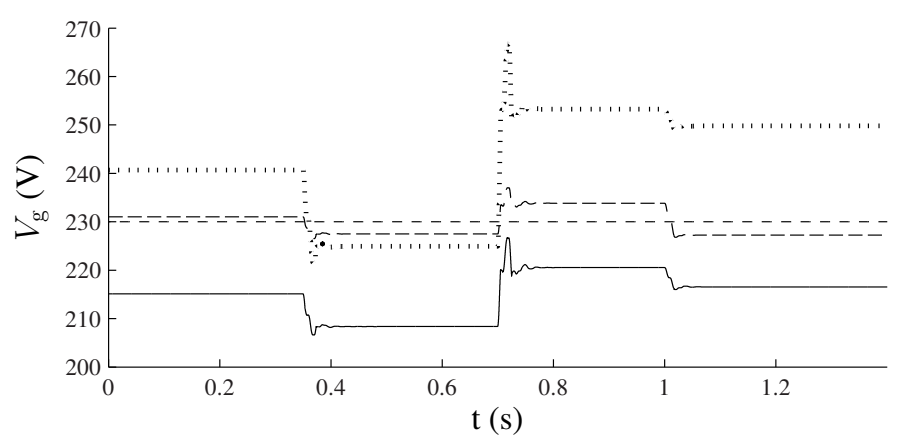

(b) Terminal voltage

Fig. 8. Grid-following trial strategy, $70 \%$ control, $I_{\mathrm{dc}, \text { nom }}: 5 \mathrm{~A}, 7.5 \mathrm{~A}$ and 10 A $(-=$ DG $1 ;---=$ DG $2 ; \cdots=$ DG $3,---=$ PCC $)$

for voltage limiting. This shows that grid-forming control is possible in grid-connected units. As in grid-connected mode, $f \approx f_{\text {nom }}=50 \mathrm{~Hz}$, the DG units operate at power-factor-one, analogous to the grid-following controllers.

In the studied case, the nominal dc-currents of the DG 


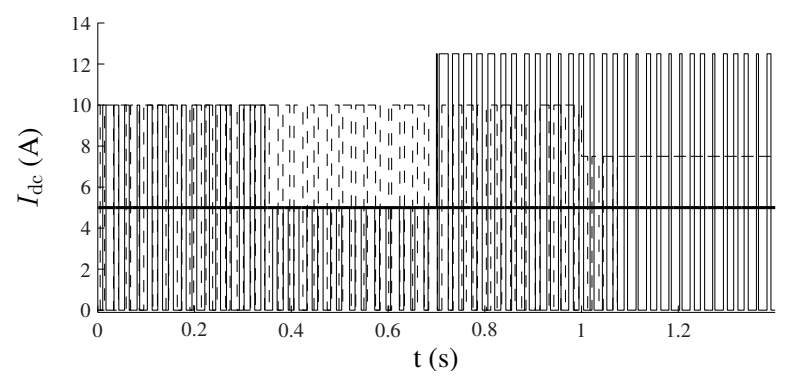

(a) Dc-input current of DG unit

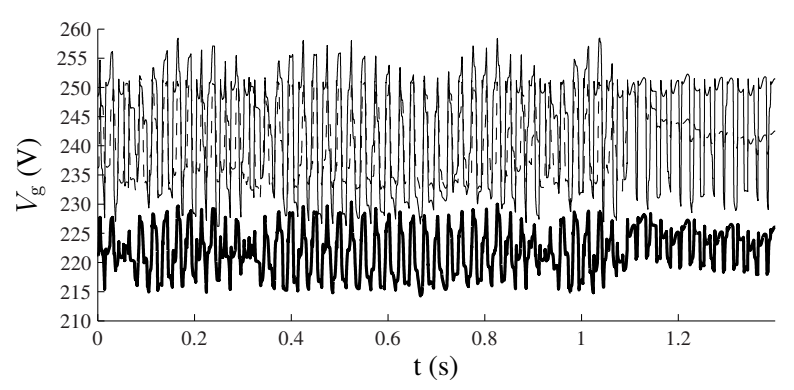

(b) Terminal voltage

Fig. 9. Grid-forming on-off control $(-=$ DG $1 ;----=$ DG $2 ;-=$ DG 3, ..$---=$ PCC in (b))

units again equal $5 \mathrm{~A}, 7.5 \mathrm{~A}$ and $10 \mathrm{~A}$ for the grid-forming units DG 1, DG 2 and DG 3 respectively. Despite the onoff oscillations, the voltage limits are met, which is shown in Fig. 9. Analogous as in the grid-following controllers, a trial strategy can be used as well.

It is concluded that grid-forming control is possible in the grid-connected microgrids. The grid-forming and gridfollowing on-off controllers have analogous results concerning voltage swings.

\section{B. VBD control}

In Fig. 10, the nominal dc-currents of the DG units equal $5 \mathrm{~A}, 10 \mathrm{~A}$ and $10 \mathrm{~A}$ for DG 1, DG 2 and DG 3 respectively (other microgrid configurations with VBD control have been studied in [27], [30]). Soft curtailment is included in these DG units by setting the constant-power band $b$ equals 0,4 and $7 \%$. In contrast to the $P / V_{\mathrm{g}}$ droop controllers, the constant-power band in VBD control allows to distinct between dispatchable and less dispatchable DG units, while still allowing the renewables to participate in the voltage control.

The droops of the controllers are set analogously as in [27], with $K_{\mathrm{P}}=-P_{\mathrm{dc}, \mathrm{nom}} / 10, K_{V}=-1 \mathrm{~V} / \mathrm{V}$ and $K_{\mathrm{Q}}=-25 \cdot 10^{-10} \mathrm{VAr} \cdot \mathrm{s}$. In the first $0.45 \mathrm{~s}$, DG 2 and 3 deliver less than $I_{\mathrm{dc} \text {,nom }}$ to the network (by using fuel intake change, storage, deviation from MPPT, load shifting) as the local voltage exceeds the constant-power bands. Although the $I_{\mathrm{dc}, \text { nom }}$ values are higher than in the previous cases, making the voltage problem more stringent, the VBD controller avoids the voltage swings and the voltage limits are met. For the case with $5 \mathrm{~A}, 7.5 \mathrm{~A}$ and $10 \mathrm{~A}$ for DG 1, DG 2 and DG 3 respectively, analogous results are obtained except that DG 2 delivers $I_{\mathrm{dc}, \text { nom }, 2}$ as the voltage does not exceed its constantpower band.

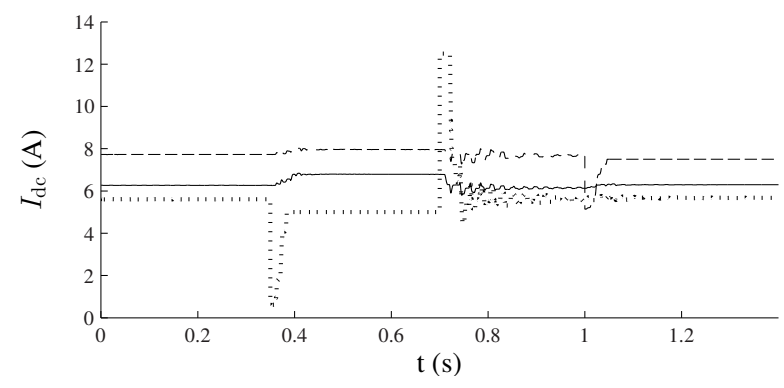

(a) Dc-input current of DG unit

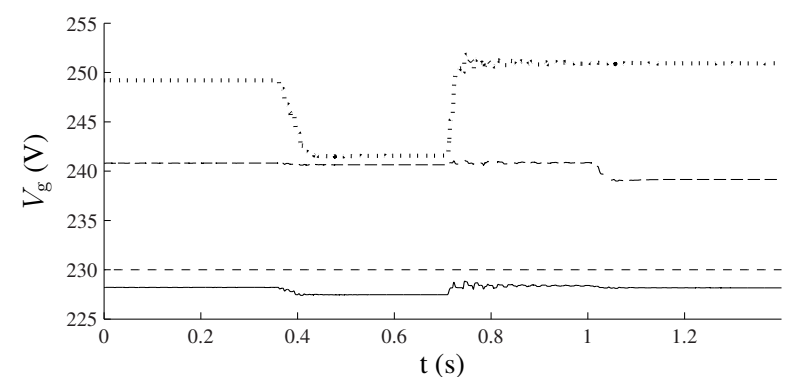

(b) Terminal voltage

Fig. 10. VBD control, first case $(-=$ DG 1 ; ---- = DG $2 ; \cdots=$ DG 3 , -.-.= PCC)

In Fig. 11, the nominal dc-currents of the DG units equal $10 \mathrm{~A}$ for all DG units and all units are considered as renewable with a large constant-power band $b=7 \%$. As the DG 1 and 2 react less on deviations of the voltage from the nominal value compared to the previous case, they deliver more power to the network. Hence, DG 3 contributes more to the voltage support compared to the previous case. The simulations show that despite the higher nominal power of the DG units compared to the on/off control and the renewable nature of all DG units, the voltage remains in the $10 \%$ limits and voltage oscillation is avoided.

VBD control with $Q / V$ droops shows analogous results as the conventional VBD control with respect to the on-off oscillations. The main difference is in the renewable energy capturing, which is analysed in the next paragraph.

\section{Captured energy}

1) Comparison between on-off and conventional VBD control: Table II summarizes the captured energy for all studied cases. DG 3 is highlighted as it is assumed as the renewable energy source most affected by voltage problems. In the gridfollowing control, when comparing the first three cases in the table, with the same $I_{\mathrm{dc} \text {,nom }}$ but with different power curtailment, the trial strategy with the $70 \%$ controller captures significantly more of the available renewable energy of DG 3. However, as stated above, finding the optimal percentage of power decrease in this method is impractical. For the other DG units, there is no significant difference in the delivered energy in the three cases. In the fourth case, with higher $I_{\mathrm{dc}, \mathrm{nom}, 1}$ and $I_{\mathrm{dc}, \text { nom,2 }}$, DG 2 delivers more energy to the network, while the captured energy of DG 3 is clearly diminished. This is especially disadvantageous if DG 3 is a renewable energy source. 


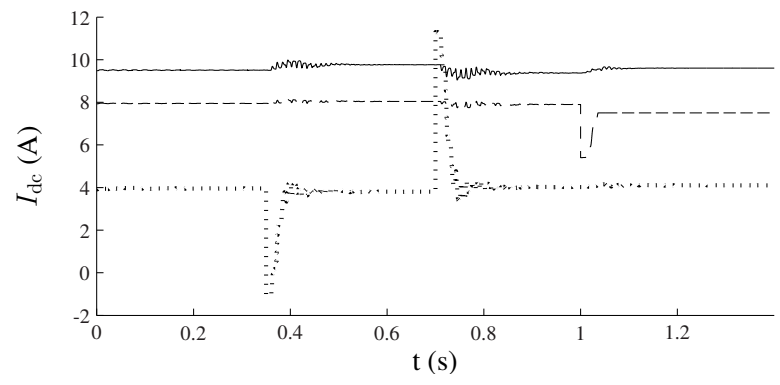

(a) Dc-input current of DG unit

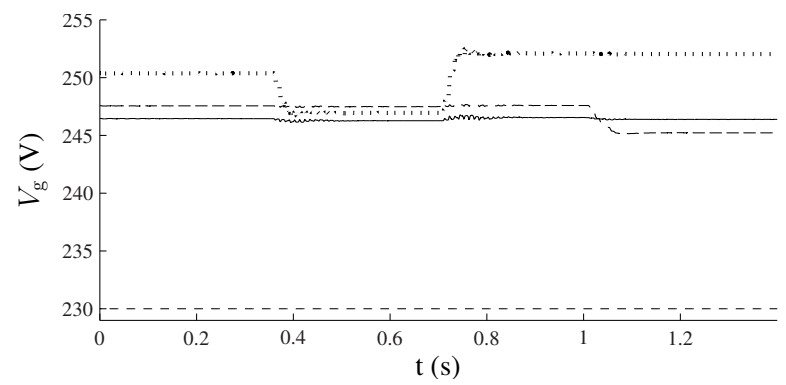

(b) Terminal voltage

Fig. 11. VBD control, second case, but all units have constant-power band of $7 \%(-=$ DG 1 ; --- = DG $2 ; \cdots=$ DG 3 , -..-- = PCC $)$

For the grid-forming on-off controllers, analogous conclusions can be made. DG 3 is clearly negatively affected if the installed power of the other units increases.

In the VBD control, for the same $I_{\mathrm{dc}, \text { nom }}$, DG 3 delivers more power to the network than in the other two control strategies. The renewable energy capturing of DG 3 is even less affected if the other units are dispatchable as they act first on the voltage changes. When all considered units are renewable energy-based, still DG 3 is affected most, because of the network configuration, but the renewable energy loss is lower than in case of the other two controllers. Also, the total renewable energy capturing by the three units is higher such that less power needs to be imported from the utility network.

2) Comparison between VBD control without and with $Q / V$ droops: In order to compare the VBD control without and with $Q / V$ droops, the network of Fig. 6 is used. All DG units are renewable with $b=7 \%$ and $I_{\mathrm{dc}, \text { nom }}=10 \mathrm{~A}$. When compared in the purely resistive network, the impact of $Q$ on the captured renewable energy is negligible. This is explained through (2) showing that a very large amount of reactive power is required to avoid the DG unit influencing the terminal voltage. In case the network of Fig. 6 has a realistic $R / X=3$, the influence of the $Q / V$ droops on the captured energy becomes larger as illustrated in Table

Although VBD control is unconventional compared to the on-off controllers, as illustrated above, a higher renewable energy capturing, less on/off oscillation and potentially a higher renewable energy source penetration (or equivalently, postponement/avoidance of investments in stronger lines for renewable energy connection) can be achieved. A drawback is that instead of a conventional on-off controller, the VBD control strategy needs to be implemented in the inverter, however, this is a one-time additional cost. By including $Q / V$
TABLE I

CAPTURED ENERGY $(\mathrm{J})$

\begin{tabular}{c||c|c|c|c} 
Grid-foll. & on-off & $50 \%$ & $70 \%$ & on-off \\
$I_{\mathrm{dc}, \text { nom }}(\mathrm{A})$ & $5 / 7.5 / 10$ & $5 / 7.5 / 10$ & $5 / 7.5 / 10$ & $10 / 10 / 10$ \\
\hline DG 1 $E[\mathrm{~J}])$ & 4896 & 4894 & 4893 & 9794 \\
DG 2 $(E[\mathrm{~J}])$ & 6849 & 6848 & 6856 & 9122 \\
DG 3 $(E[\mathrm{~J}])$ & 5775 & 6842 & 7991 & 4002
\end{tabular}

\begin{tabular}{c||c|c|c|c}
\hline \hline Grid-form. & on-off & on-off & on-off & \\
$I_{\text {dc,nom }(\mathrm{A})}$ & $5 / 7.5 / 10$ & $5 / 10 / 10$ & $10 / 10 / 10$ & \\
\hline DG 1 $(E[\mathrm{~J}])$ & 5920 & 5963 & 8526 & \\
DG 2 $(E[\mathrm{~J}])$ & 9661 & 10051 & 8081 & \\
DG 3 $(E[\mathrm{~J}])$ & 5302 & 5208 & 4105 & \\
\multicolumn{5}{|l|}{} \\
\hline \hline Grid-form. & VBD & VBD & VBD & VBD-RE \\
$I_{\text {dc,nom }(\mathrm{A})}$ & $5 / 7.5 / 10$ & $5 / 10 / 10$ & $10 / 10 / 10$ & $10 / 10 / 10$ \\
\hline DG 1 $(E[\mathrm{~J}])$ & 7087 & 6780 & 7942 & 11539 \\
DG 2 $(E[\mathrm{~J}])$ & 7978 & 9166 & 8708 & 9402 \\
DG 3 $(E[\mathrm{~J}])$ & 6691 & 6576 & 6279 & 4741 \\
\hline
\end{tabular}

droops as well, the energy capturing can further be increased.

TABLE II

CAPTURED ENERGy $E(\mathrm{~J})$ : VBD VERSUS VBD With $Q / V$ DROOPS

\begin{tabular}{c||c|c|c|c}
\hline \multicolumn{1}{l||}{} & \multicolumn{2}{c|}{ R-grid } & \multicolumn{2}{c}{$R / X=3$} \\
& VBD & VBD+Q/V & VBD & VBD $+Q / V$ \\
\hline \hline$E_{1}$ & 10760 & 11146 & 11813 & 11967 \\
$E_{2}$ & 10303 & 10817 & 9233 & 9474 \\
$E_{3}$ & 4486 & 4663 & 4920 & 5643 \\
\hline
\end{tabular}

\section{CONClusion}

In this paper, the behaviour of grids with distributed renewable energy sources is studied. The on-off oscillations and renewable energy capturing are studied for grids driven with on-off and droop controllers. In case the units are gridfollowing and equipped with conventional on-off control, large voltage swings and renewable energy loss are observed. Hence, instead of completely shutting down the unit, the delivered power should be a function of the terminal voltage, while still communication should be avoided. For this, a control strategy is discussed that does not completely shut down the DG units but lowers their power with a certain percentage.

The paper shows that using grid-forming units in the gridconnected system, with on-off controllers, is possible as well and leads to analogous voltage problems. Therefore, a variant of $P / V_{\mathrm{g}}$ droop control, the grid-forming VBD control, is used. This control strategy avoids voltage limit violation without the on-off swings that occurred in the other cases. It also retrieves a higher renewable energy capturing. Important is that VBD control does not require communication and automatically gives priority injection to the renewable energy sources, in contrast to the conventional $P / V_{\mathrm{g}}$ droops (which also avoid the on-off oscillations).

Finally, in this paper, the VBD control is extended with $Q / V$ droops. By consuming reactive power, the impact of the 
DG unit on the terminal voltage is lowered. Relying on this, with $Q / V$ droops, the renewable energy capturing is increased. However, in rural networks with a high line resistance, the impact of the reactive power consumption on the network losses can prove it better to use the VBD control without $Q / V$ droops.

\section{ACKNOWLEDGEMENTS}

This work is financially supported by the FWO-Vlaanderen (Research Foundation - Flanders, Belgium). T. Vandoorn thanks the FWO for the Fellowship received. The research of J. D. M. De Kooning is funded by the Special Research Fund (BOF) of Ghent University (Belgium). This research has been carried out in the frame of the Inter-university Attraction Poles Programme initiated by the Belgian Science Policy Office.

\section{REFERENCES}

[1] F. Blaabjerg, R. Teodorescu, M. Liserre, and A. V. Timbus, "Overview of control and grid synchronization for distributed power generation systems," IEEE Trans. Ind. Electron., vol. 53, no. 5, pp. 1398-1408, Oct. 2006.

[2] P. Chiradeja and R. Ramakumar, "An approach to quantify the technical benefits of distributed generation," IEEE Trans. Energy Convers., vol. 19, no. 4, pp. 764-773, Dec. 2004.

[3] P. Dondi, D. Bayoumi, C. Haederli, D. Julian, and M. Suter, "Network integration of distributed power generation," Journal of Power Sources, vol. 106, pp. 1-9, 2002.

[4] I. Wasiak, M. C. Thoma, C. E. T. Foote, R. Mienski, R. Pawelek, P. Gburczyk, and G. M. Burt, "A power-quality management algorithm for low-voltage grids with distributed resources," IEEE Trans. Power Del., vol. 23, no. 2, pp. 1055-1062, Apr. 2008.

[5] Y. Li, D. M. Vilathgamuwa, and P. C. Loh, "Microgrid power quality enhancement using a three-phase four-wire grid-interfacing compensator," IEEE Trans. Ind. Appl., vol. 41, no. 6, pp. 1707-1719, Nov./Dec 2005.

[6] S. Barsali, M. Ceraolo, P. Pelacchi, and D. Poli, "Control techniques of dispersed generators to improve the continuity of electricity supply," in Proc. IEEE PES Winter Meeting, 2002, pp. 789-794.

[7] R. H. Lasseter, A. Akhil, C. Marnay, J. Stephens, J. Dagle, R. Guttromson, A. Meliopoulous, R. Yinger, and J. Eto, "The CERTS microgrid concept, white paper on integration of distributed energy resources," in California Energy Commission, Office of Power Technologies - U.S. Department of Energy, LBNL-50829, http://certs.lbl.gov, Apr. 2002.

[8] R. H. Lasseter and P. Paigi, "Microgrid: A conceptual solution," in Proc. IEEE Power Electron. Spec. Conf. (PESC 2004), Aachen, Germany, 2004.

[9] N. Lidula and A. D. Rajapakse, "Microgrids research: A review of experimental microgrids and test systems," Renewable and Sustainable Energy Reviews, vol. 15, no. 1, pp. 186-202, 2011.

[10] A. Mehrizi-Sani and R. Iravani, "Potential-function based control of a microgrid in islanded and grid-connected modes," IEEE Trans. Power Syst., vol. 25, no. 4, pp. 1883-1891, Nov. 2010.

[11] H. Farhangi, "The path of the smart grid," in IEEE Power \& Energy Magazine, Jan./Feb. 2010, vol. 8, no. 1, pp. 18-28.

[12] A. G. Tsikalakis and N. D. Hatziargyriou, "Centralized control for optimizing microgrids operation," IEEE Trans. Energy Convers., vol. 23, no. 1, pp. 241-248, Mar. 2008.

[13] F. Katiraei, M. R. Iravani, and P. W. Lehn, "Micro-grid autonomous operation during and subsequent to islanding process," IEEE Trans. Power Del., vol. 20, no. 1, pp. 248-257, Jan. 2005.

[14] A. Vaccaro, M. Popov, D. Villacci, and V. Terzija, "An integrated framework for smart microgrids modeling, monitoring, control, communication, and verification," Proc. IEEE, vol. 99, no. 1, pp. 119 - 132, Jan. 2010

[15] C. Sao and P. Lehn, "Control and power management of converter fed microgrids," IEEE Trans. Power Syst., vol. 23, no. 3, Aug. 2008.

[16] L. R. Limongi, R. Bojoi, G. Griva, and A. Tenconi, "Digital currentcontrol schemes," IEEE Ind. Electr. Magazine, vol. 3, no. 1, pp. 20-31, Mar. 2009.

[17] M. P. Kazmierkowski and L. Malesani, "Current control techniques for three-phase voltage-source PWM converters: A survey," IEEE Trans. Ind. Electron., vol. 45, no. 5, pp. 691-703, Oct. 1998.
[18] B. Meersman, B. Renders, L. Degroote, T. Vandoorn, and L. Vandevelde, "Three-phase inverter-connected DG-units and voltage unbalance," Electric Power Systems Research, vol. 81, no. 4, pp. 899-906, 2011.

[19] M. C. Chandorkar, D. M. Divan, and R. Adapa, "Control of parallel connected inverters in standalone ac supply systems," IEEE Trans. Ind. Appl., vol. 29, no. 1, pp. 136-143, Jan./Feb. 1993.

[20] J. M. Guerrero, J. Matas, L. García de Vicuña, M. Castilla, and J. Miret, "Wireless-control strategy for parallel operation of distributed-generation inverters," IEEE Trans. Ind. Electron., vol. 53, no. 5, pp. 1461-1470, Oct. 2006.

[21] J. A. Peças Lopes, C. L. Moreira, and A. G. Madureira, "Defining control strategies for microgrids in islanded operation," IEEE Trans. Power Syst., vol. 21, no. 2, pp. 916-924, 2006.

[22] H. Laaksonen, P. Saari, and R. Komulainen, "Voltage and frequency control of inverter based weak LV network microgrid," in 2005 International Conference on Future Power Systems, Amsterdam, Nov. 18, 2005.

[23] A. Engler, O. Osika, M. Barnes, and N. Hatziargyriou, DB2 Evaluation of the local controller strategies. www.microgrids.eu/micro2000, Jan. 2005.

[24] J. A. Peças Lopes, N. Hatziargyriou, J. Mutale, P. Djapic, and N. Jenkins, "Integrating distributed generation into electric power systems: A review of drivers, challenges and opportunities," Electric Power Systems Research, vol. 77, pp. 1189-1203, 2007.

[25] SMA Solar Technology. (2010, Jun.) SMA inverters as grid managers.

[26] M. H. J. Bollen and N. Etherden, "Overload and overvoltage in lowvoltage and medium-voltage networks due to renewable energy - some illustrative case studies," in IEEE PES Innovative Smart Grid Technologies (ISGT 2011), Manchester, UK, Dec. 5-7, 2011.

[27] T. L. Vandoorn, B. Meersman, L. Degroote, B. Renders, and L. Vandevelde, "A control strategy for islanded microgrids with dc-link voltage control," IEEE Trans. Power Del., vol. 26, no. 2, pp. 703-713, Apr. 2011.

[28] T. L. Vandoorn, B. Renders, L. Degroote, B. Meersman, and L. Vandevelde, "Active load control in islanded microgrids based on the grid voltage," IEEE Trans. on Smart Grid, vol. 2, no. 1, pp. 139-151, Mar. 2011.

[29] M. H. J. Bollen and F. Hassan, Integration of Distributed Generation in the Power System, ser. IEEE Press Series on Power Engineering. John Wiley \& Sons, 2011.

[30] T. L. Vandoorn, B. Meersman, J. D. M. D. Kooning, and L. Vandevelde, "Controllable harmonic current sharing in islanded microgrids: DG units with programmable resistive behavior towards harmonics," IEEE Trans. Power Del., vol. 27, no. 2, pp. 831-841, Apr. 2012.

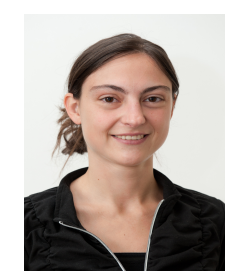

Tine Vandoorn (S'09) was born in Torhout, Belgium in 1985. She received the M.S. in electromechanical engineering from Ghent University, Ghent, Belgium, in 2008. In 2008, she joined the Electrical Energy Laboratory (EELAB) of Ghent University where she is currently pursuing the Ph.D. degree in electromechanical engineering. Her present research interests include electric power systems, voltage and power control of DG units, management of microgrids and smart microgrids. In 2009, she was awarded a grant as Ph.D. fellow of the Research Foundation - Flanders (FWO)

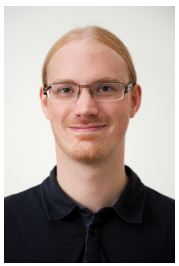

Jeroen De Kooning (S'09) was born in Kapellen, Belgium in 1987. He received the M.S. degree in electromechanical engineering from Ghent University, Belgium, in 2010. Since then, he is with the Electrical Energy Laboratory (EELAB) of Ghent University and is currently pursuing the Ph.D. degree in electromechanical engineering. His present research interests include wind energy systems, control of power-electronic converters and brush-less ac drives. 


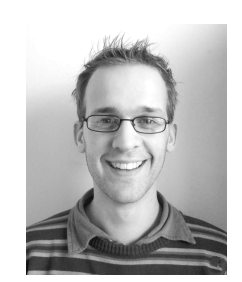

Bart Meersman (S'07) was born in Sint-Niklaas, Belgium in 1983. He received the M.S. and Ph.D degree in electromechanical engineering from Ghent University, Belgium, in 2006 and 2012, respectively. $\mathrm{He}$ is with the Electrical Energy Laboratory (EELAB), Ghent University, since 2006, where he is a postdoctoral researcher in electrical power engineering. His research activities are in the field of electric power systems, renewable energy applications, power electronics and digital control of power quality.

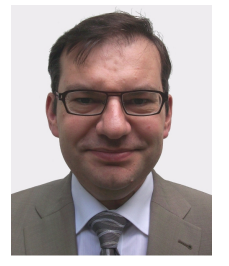

Lieven Vandevelde (M05 - SM07) was born in Eeklo, Belgium, in 1968. He graduated as Master of Electromechanical Engineering and received the $\mathrm{Ph} . \mathrm{D}$. degree in electromechanical engineering from Ghent University, Belgium, in 1992 and 1997, respectively. He is with the Electrical Energy Laboratory (EELAB), Ghent University, where he has been a professor in electrical power engineering since 2004. His research and teaching activities are in the field of electric power systems, electrical machines and (computational) electromagnetics. 